\title{
OPTIMISED INTAKE STROKE ANALYSIS FOR FLAT AND DOME HEAD PISTONS
}

\author{
A. A. Ishola ${ }^{1, *}$, A. M. Alsheri' ${ }^{2}$ and A. I. Musa ${ }^{3}$ \\ 1AEROSPACE ENGINEERING DEPARTMENT, UNIVERSITY OF HERTFORDSHIRE, UNITED KINGDOM \\ ${ }^{2}$ AUTOMOTIVE ENGINEERING DEPARTMENT, UNIVERSITY OF HERTFORDSHIRE, UNITED KINGDOM \\ 3 Mechanical Engineering Department, Moshood Abiola Polytechnic, Abeokuta, Ogun State. Nigeria. \\ E-mail addresses: 1 aa.ishola@yahoo.co.uk,2am.alsheri@yahoo.com, ${ }^{3}$ kunlemusa@yahoo.com
}

\begin{abstract}
This research exerts are suitable for the automobile industry in understanding the performance characteristics optioned between flat head and dome head pistons in engine design. This study was carried out to analyze the optimization parameters for effective and efficient flow characteristics of air-fuel mixture at the intake port of the combustion chamber of an internal combustion engine. $A$ unique and industrial standard CFD software, STAR-CCM V8, was used to model both geometry for flat head and dome head pistons which was developed with precise dimensions of a 1.8L gasoline engine. $A$ planar 3-D model approach was adopted for simplified static CAD modeling and also to reduce the solver processing time. The piston models were meshed using tetrahedral mesh of base size $0.001 \mathrm{~m}$. The boundary and physics conditions were applied to simulate the actual intake stroke process for normal operating conditions and initial conditions. The extracted results were validate and comparisons developed to analyze the various optimization parameters for performance characteristics of the two pistons.
\end{abstract}

Keywords: optimized intake stroke, CFD analysis, flat and dome head pistons, internal combustion engine, CAD modelling

\section{NOMENCLATURE}

$\rho=$ density

$\overrightarrow{\boldsymbol{U}}=$ uniform velocity

$\eta \dot{n}=$ dynamic viscosity

$\Delta \boldsymbol{p}=$ pressure gradient

$\mathrm{F}=$ external fluid force.

\section{INTRODUCTION}

Advancement in internal combustion engine design has taking new dimensions in optimization methods of improving engine performance. Over the years simulation of optimization characteristics has followed dynamic changes in conducting laboratory experiments. In recent times Computational Fluid Dynamics (CFD) analysis has been the fore front of advanced research techniques in determining air-fuel flow profile suitable for internal combustion engines. This involves the use of computer algorithms to model and simulate flow characteristics which literarily predict the occurrences in the combustion chamber that are normally difficult to witness in operation. CFD optimization methods are designed for aerodynamics problems and computing this experimentally normally takes several hours or even days to derive results. Computational time could be expensive and difficulties could arise as a result of inaccurate modelling attributes. CFD codes are generated on adjoint solvers and concatenation of models are computationally expensive in terms of CPU effort in geometrical processing. Dealing with multi-objective optimization is of great importance in design of internal combustion engines.

Turbulence modelling is described as the function of using a model to analyse turbulence. Turbulence is the spontaneous and erratic behaviour of fluids where indiscriminate changes occur in fluid parameters within a specified boundary.

Turbulence modelling has been one of the most difficult to analyze flow problems in the engineering field. There are numerous turbulence models developed over centuries to describe the turbulent flow. Navier-Stokes (NS) equations is one of the common and unsolved turbulence model in the world. The closure problem arising from many unknown terms in the equation has been unsolved for many years. Reynolds Averaged Navier-Stokes (RANS) adopts the principle of timeaveraging to simplify Navier-Stokes equation. Among the continuum categories of turbulence models are zeroequation models, one-equation models, two-equation 
models, however, the most common and simplified models include:

- $\quad$ K-epsilon (k-e) model

- K-omega (k-w) model

- $\quad$ Spallart-Allmaras

- Reynolds stress equation model

In understanding the principle of operation for conducting optimization regarding the combustion chamber it requires the knowledge of turbulent flow characteristics and parameters. Equations have been developed to assign variables in CFD experimentation using one of the common turbulence models. An important and unfortunate aspect of CFD research is the limitations of complete description of all characteristics occurring in the combustion chamber but it is also important to note the rapid change in effects of simulation parameters. The problem of heat interaction with the fluid has been considered in this experiment also to include the heat transfer between the cylinder walls and the thermo-fluid. Engine temperature adopted for CFD experiment is based on assumptions.

\subsection{Aims and Objectives}

This research is aimed at analyzing the optimized characteristics of the intake stroke for flat and dome head pistons used in internal combustion engines with the application of a unique CFD software, STAR-CCM V8. The visualized patterns and extracted parameters of the solver process will enable the better understanding of the flow characteristics at the intake stroke with regards to the choice of piston with optimum intake performance as well as set parameters for variables in piston performance analysis.

\section{TECHNICAL BACKGROUND}

The most important part of an engine design is the combustion chamber and apparently all other subsystems are developed around it. Combustion process involves igniting a highly charged air-fuel mixture within the engine cylinder causing the gas to expand and forcing the piston to rotate the crank-shaft. This phenomenon sets the piston to move between the TDC and BDC in a reciprocating form. The two main types of this engine classification are the two-stroke and four-stroke engines. The four stroke engine operates in a cycle starting with intake then compression and expansion to exhaust strokes.

At intake stroke, the inlet valve is opened and the exhaust valve is closed at TDC [1]. As the piston moves downwards, fresh charge enters and fills the combustion chamber. In the last decade, researchers have been concerned with optimising internal combustion engine performances using CFD tools. Very recent experiments such as that of Mark Fogleman et al [2] presented analysis on turbulence modelling of internal combustion engines using Proper Orthogonal Decomposition (POD) method to both CFD and particle imaging velocimetry data of simplified motored engine flows. Ugur Kesgin, [3], adopted the use of CFD to determine overall engine performance using design and operational variables of inlet and exhaust systems. Ugur's models were basically adopting zero dimensional phenomena within the engine cylinder and one dimensional phenomena at the engine inlet and exhaust considering valve timing which was tuned, valve diameter and diameter of exhaust manifold.


Figure 1: Intake stroke of an internal combustion engine (flat and dome head) 
Stefan Gundmalm, [4], had made significant progress on CFD modeling of a four stroke engine with the use of Open FOAM software which is also an industrially adopted software to simulate internal combustion processes. This model was able to maintain a platform for comparison in the areas of pressure distribution, turbulent kinetic energy as well as heat transfer in the combustion.

\section{OPTIMIZATION AND CFD PARAMETRIZATION}

Defining the parameters that determine a specified area of improvement is termed as optimization. The set parameters for optimizing the combustion chamber are numerous and impossible to capture as a whole. Some of them include inlet velocity, engine temperature, density, pressure, turbulent viscosity etcetera. The variables involved in optimized performance of internal combustion engines can be streamlined into a particular section of improvement. Establishing the relationship between objective functions and control variables is paramount to a parameterization [5]. To achieve this aim, the important basic steps required to perform CFD analysis include:

1. Pre-processing. Involves geometrical modelling of the domain for which CFD is to be conducted. Geometrical modelling technique is performed directly or obstructively. Direct modelling is simply presenting a CAD model of the domain or geometry using suitable dimensions or scaling. Abstractive modelling is a technique that materialises the concentrated domain using planar principle. Planar modelling adopts logic insight into cut-section plane of the actual model. This platform is very useful for simplifying the geometry and time required for complete geometrical processing. Preprocessing also involves mesh generation by using suitable finite element models. Finite volume models are also used for three dimensional modelling. However, accuracy of results can depend on meshing technique adopted for simulation. Boundary conditions are important in CFD runs where the condition variables are computed and boundary characteristics are defined for the geometry.

2. Computation: This is the solver operations performed by the computer. Iterative methods are used while the CPU calculates and computes the solution. Parameters of computation include stability, convergence and consistency. Solvers can be performed using implicit or explicit methods. Depending on the solution control, convergence is the determinant on attaining an acceptable solution.

3. Post-processing. Experiments are performed to achieve results. Therefore, post-processing is the extraction of results from CFD runs but results are presented in visual modes and colour legend for analysis.

\subsection{Optimized Intake Stroke Analysis Using Star-Ccm For Flat And Dome Head Pistons}

STAR-CCM is industrially recognized software used to model flow problems. It is used to simulate fluids such as viscous fluids, gases, solids also the conditions such as boundary conditions, physics conditions etc. High speed computers are used to perform calculations which usually generate reliable solutions. In this report, the aim virtually stresses the research into the modelling of turbulent flows. Though, more time is dedicated to the geometrical processing of the problem including meshing as well as running the simulation. STAR-CCM is programmed with turbulence models which will be used to analyze the flow of air at the intake stroke. Temperature phenomenon is accustomed with internal combustion engines. Heat flux generated from the combustion process and surrounding heat have effect on gases. This analysis include the effect of temperature variables at the intake.
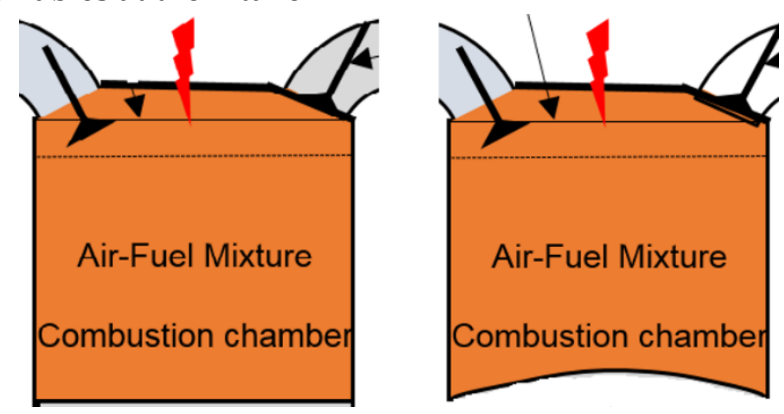

Figure 2: Flat and dome head piston models to be analyzed

\subsubsection{Governing Equations}

Mass continuity equation for incompressible flow;

$$
\nabla . U=0
$$

Starting with the equations of motion, the Navier-Stokes equation is given as;

$$
\rho \frac{d \vec{U}}{d t}+\rho \vec{U} \cdot \nabla \vec{U}=-\Delta p+F+\eta^{\prime} \nabla^{2} \vec{U}
$$

Air enters the combustion chamber at high speeds and are sometimes supersonic in nature therefore, it is important to present assumed values for the inlet velocity. The turbulent model adopted for performing the analysis is k-epsilon (k-e) model. Before using k-e model it is important to state the variable parameters for analysis. 


\subsubsection{Assumptions}

- $\quad$ Inlet velocity $(\mathrm{u})=15 \mathrm{~m} / \mathrm{s}$

- $\quad$ Engine Temperature $\left(\mathrm{T}_{\mathrm{E}}\right)=1273 \mathrm{~K}$

- $\quad$ Combustion Temperature $\left(\mathrm{T}_{\mathrm{C}}\right)=2773 \mathrm{~K}$

- $\quad$ Pressure $\left(\mathrm{P}_{\mathrm{atm}}\right)=101210 \mathrm{pa}$

- Density $(\rho)=1.205 \mathrm{kgm}^{-3}$

- $\quad$ Kinematic viscosity $(v)=1.544 \times 10^{-5} \mathrm{~m}^{2} \mathrm{~s}^{-1}$

- Dynamic viscosity $\mu$ is given by:

$$
\mu=v \times \rho=1.8605 \times 10^{-5} \mathrm{kgm}^{-1} \mathrm{~s}^{-1}
$$

- $\quad$ Specific Heat $\left(\mathrm{H}_{\mathrm{s}}\right)=1007 \mathrm{JKg}^{-1} \mathrm{~K}^{-1}$

- $\quad$ Diameter of intake valve $(\mathrm{L})=0.04 \mathrm{~m}$

- Reynolds Number:

$$
(R e)=\frac{u L}{v}=38860.10363
$$

- $\quad$ Length Scale;

$$
(l)=0.07 \times L==2.8 \times 10^{-3} \mathrm{~m}
$$

- Turbulence Intensity

$$
(I)=0.16(R e)^{-0.125}=0.04276958
$$

- Turbulence Kinetic Energy

$$
k=\frac{2}{3}(I u)^{2} 0.61736747 \mathrm{~J} / \mathrm{kg}
$$

- Turbulence Dissipation Rate:

$$
=\frac{\left(C_{\mu}\right)^{0.75} k^{1.5}}{l}=28.468929 \mathrm{~m}^{2} / \mathrm{s}^{3}
$$

3.2 Turbulence Flow Model at Intake Stroke for Flat and Dome Head Pistons with Temperature Effects

\subsubsection{Geometry Processing}

A three dimensional planar model is used to represent the combustion chamber. A relative thickness of $0.002 \mathrm{~m}$ displays the opening of the valve and piston motion down the cylinder. Valve angle is designed at $30^{\circ}$ from valve face and opening distance of $0.005 \mathrm{~m}$. The piston bore is $80 \mathrm{~mm}$ while the stroke is $66 \mathrm{~mm}$.

\subsubsection{Mesh generation}

Volumetric meshing technique is adopted while a suitable mesh type for simulation is polyhedral mesh. Polyhedral mesh presents good volume mesh and mesh concentration is used at the relative boundaries. A base size of $0.005 \mathrm{~m}$ is used for mesh size with relative density factor of 1.1.


Figure 3: Combustion chamber geometry for both piston models
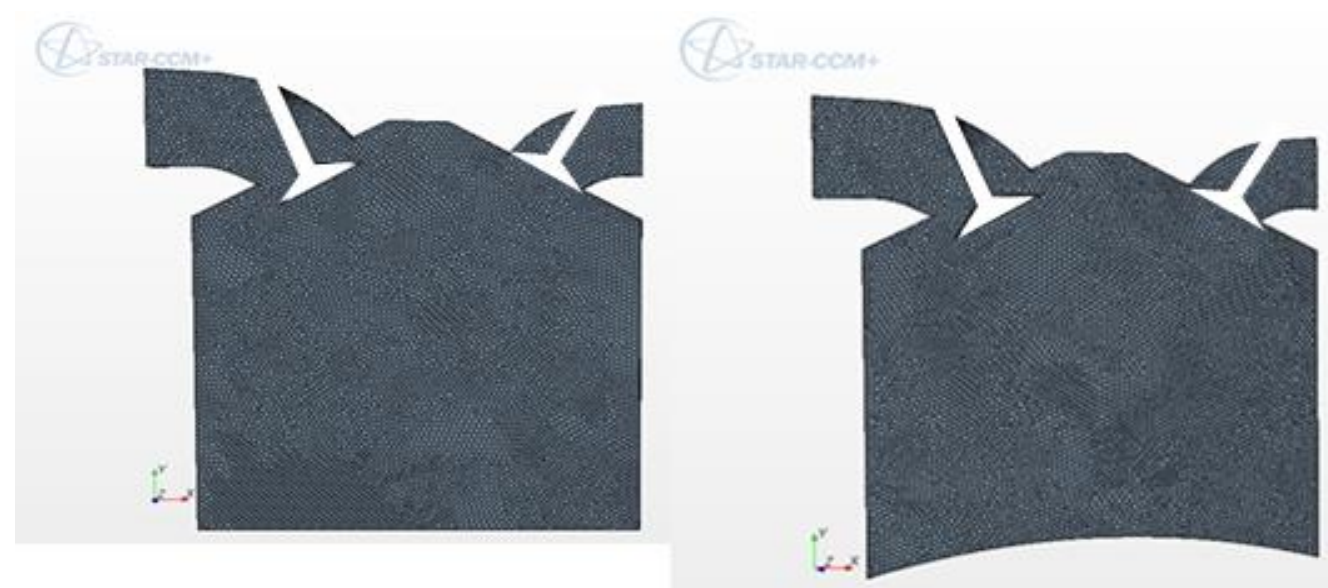

Figure 4: Infinitesimal mesh for both piston models 


\subsubsection{Physics and Boundary Conditions}

It is important to select appropriate physics condition by specifying the turbulence model and values of the state variable. The options selected for physics model are as follows;

- "Three dimensional" The model was developed in three dimensions.

- "Steady" The air-fuel mixture has a steady flow characteristics.

- "Gas" The mixture is in gaseous state.

- "Constant density" The mixture's density does not change with time.

- $\quad$ "Segregated flow" Intake stroke is characterized by as segregated flow phase index.

- "Turbulent" Intake air-fuel mixture is turbulent with a supersonic flow rate.

- "K-Epsilon" The K-e turbulence modeling is selected for the analysis.

- $\quad$ "Segregated vortex temperature" The temperature of the analysis is considered.

The boundary conditions used to simulate the flow include;

- "velocity inlet" for the intake port

- "Wall" which was assumed the exhaust valve is closed with temperature 2773K.

- "Wall" for the cylinder walls and piston head with temperature of $1273 \mathrm{~K}$.

- "No-slip condition" for the Fluid-Wall contact.

\section{RESULTS AND ANALYSIS}

Post-processing: Extraction of results after the computation is completed. This is a visual display of variable functions about the geometry.

Figure 5 presents the pressure distribution within the combustion chamber for flat and dome head pistons. In the flat head piston model, pressure is expectedly low at the inlet and extremely high at the boundary layer between the piston head and the walls of the cylinder. This high pressure relates the expansion of the hot airfuel mixture which relatively improves the event of swirl. In the dome head piston model, localized areas of pressure drop can be spotted and this is imminent with shape of the piston. The curved area of the piston-head creates a shear layer of compensated volume drop. This results to the decrease in swirl movement of the air-fuel mixture. The molecules experience bounded regions dedicated to no-slip conditions at the boundary surfaces resulting to pressure drop. Cylinder wall temperature however limits the possibility of cooling the gas whereas the mixture is dense presenting a rich mixture for combustion. This advantage of dome head pistons promotes a rich mixture that delivers more power during combustion process.

Cold air-fuel molecules at the valve opening are highly dense which is suitable for the volumetric molecular flow. The velocity of flow measured at the inlet is about 15 meters per second and deflection occurs when the molecules come in contact with the 30 degrees valve neck. As presented in Figure 6, the charge experiences high speed flow due to constriction.

In combustion chamber analysis, the peak burned gas temperature is about $2500^{\circ} \mathrm{C}(2773 \mathrm{~K})$ and surrounding temperature about $1000^{\circ} \mathrm{C}$ (1273K) [6]. This high temperature induces turbulence formed by squish.

Expansion of the charge entering the combustion chamber results into divergence. This rapidly increases the speed at the valve tips and port edges causing a partial vacuum at the clearance volume and as air tries to fill up the space a spiral motion is generated which then expands rapidly. This phenomenon is known as vortex shedding which can be viewed in figure 8 .

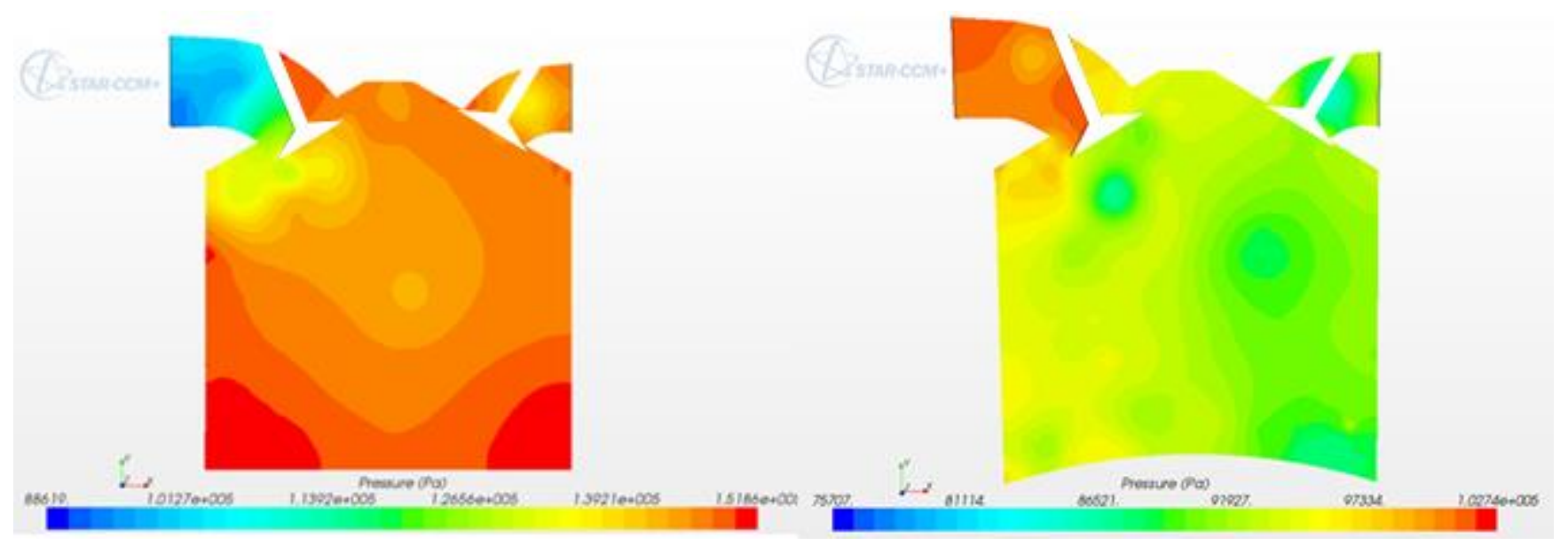

Figure 5: Pressure distribution for flat and dome piston models 




Figure 6: Velocity stream around the intake valve port

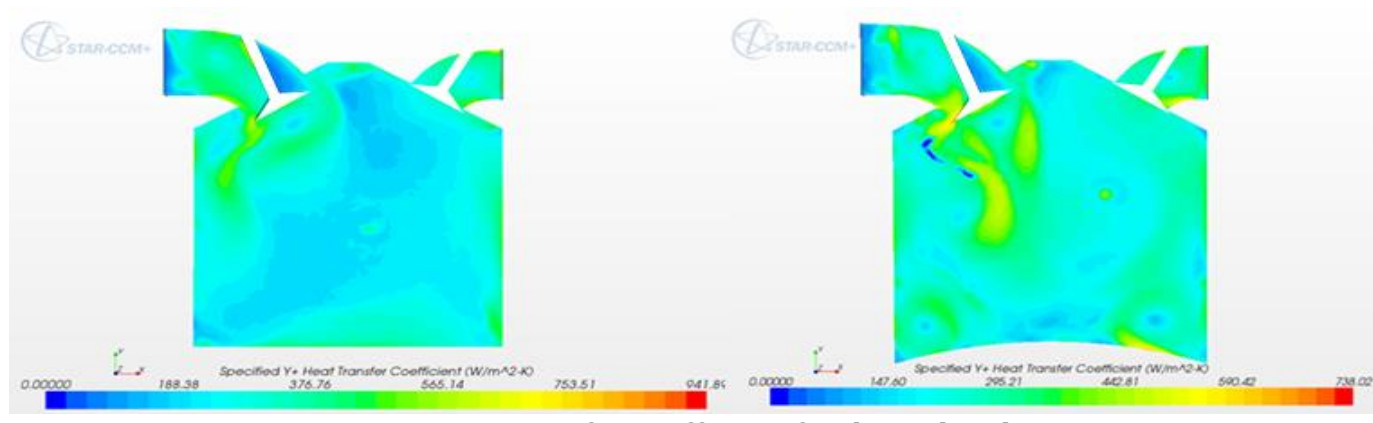

Figure 7: Heat transfer coefficient for dome-head piston

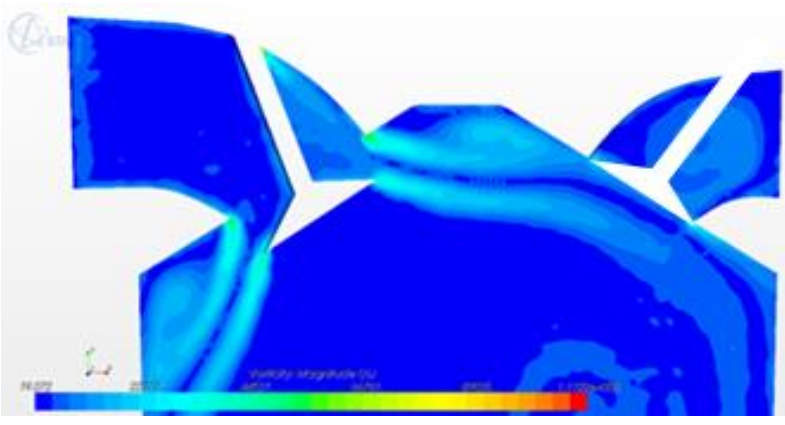

Figure 8: Vortex shedding around the valve port

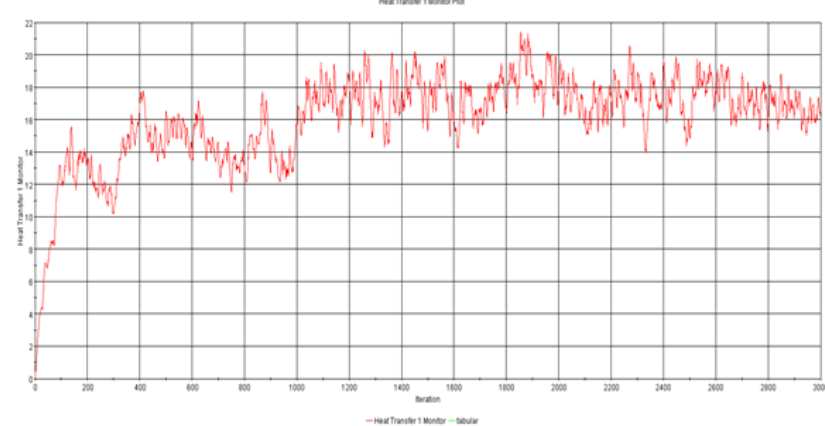

Figure 9: Turbulence Intensity monitor for intake stroke of an internal combustion engine

\subsection{Turbulence Flow Model at Intake Stroke of Flat Head Piston Without Temperature Effects}

Running the simulation for flat head piston without temperature effect, meaning that there is no surrounding temperature. This condition is termed initial phase model describing initial conditions of the model. The pressure of the charge is high at inlet port and rapidly drops is it fills the chamber forming layers of dense molecules. This analysis explains the formation of rich mixture at startup.

Nigerian Journal of Technology,



Figure 10: Pressure distribution within the combustion chamber

Figure 11 describes supersonic speeds of velocity stream at the inlet port. The charge in boundary with cool wall surface reduces speed at a steady state which requires a probe for steady-state conditions. The charge then forms a turbulent flow at the center of the combustion chamber ready to be compressed. This profile experiences an optimum degree of turbulence which is induced by inlet flow configuration.



Figure 11: Relative velocity distribution within the combustion chamber

Vol. 36, No. 2, April 2017 




Figure 12: Turbulent kinetic energy of the flat head piston model

\section{DISCUSSIONS AND CONCLUSION}

CFD simulation on STAR-CCM presented good visual display of air flow analysis at the intake stroke in the combustion chamber. However, models with temperature effects are most suitable for analysing the combustion chamber. The piston models without temperature is used to differentiate and conduct comparison between the models with temperature effect. Heat transfer is very important in piston design and the process of combustion is mainly to generate heat. Heat variation across the cylinder walls and the effect it has on gases within the combustion chamber are necessary to optimise the cooling rate of the cylinders. Comparison between the flat head and dome head pistons with temperature effects as displayed in table 1 shows that the flat head piston developed a slightly higher pressure than the dome head piston. However, the total temperature in the dome head piston is almost fifteen percent (15\%) higher than that of the flat head piston. A similar characteristics follow for the heat transfer coefficient having a difference of $25.28 \mathrm{~W} / \mathrm{m}^{2}-\mathrm{K}$. This shows that heat transfer on flat head piston is at a faster rate than in dome head type.

Air-fuel mixture velocity in flat head piston model is about 35 percent higher than in dome type which is significant to the effect of surface-to-volume ratio. The dome head obviously has more surface area than that of flat type. Thus, a wedge combustion chamber design is better optimized with a flat type than a dome type piston. Wall shear stress is significantly higher in dome piston taken into account the surface curvature which improves turbulence. The charts below expresses the relationships between the flat and dome pistons for the parameters.

The swirl effect about the valve opening is very significant and in both cases shows a directional dispersed flow pattern. This is due to the heat transfer from the cylinder walls to the fresh air intake which is cooler. The cool air absorbs heat from the surrounding surfaces and the air molecules expand with high velocities and sporadic movements. The boundary condition at the walls is no slip therefore, fluid molecules cannot flow across the boundaries.

Comparing the flat head models i.e. with and without heat, it is imminent that the pressure profile dropped significantly and apparently a pressure slightly higher at the inlet duct of the model without heat when compared to the combustion chamber. Continuous and streamlined flow circulation is also observed within the combustion chamber which indicates a cold start.

Table 1: Results analysis of variables for optimized

\begin{tabular}{|c|c|c|c|c|}
\hline \multicolumn{5}{|c|}{ parameters } \\
\hline \multirow{2}{*}{$\begin{array}{l}\begin{array}{l}\text { Optimized } \\
\text { parameters }\end{array} \\
\text { Pressure }(\mathrm{Pa})\end{array}$} & \multicolumn{2}{|c|}{ Operating condition } & \multicolumn{2}{|c|}{ Initial conditions } \\
\hline & $\begin{array}{l}1.5186 x \\
10^{5}\end{array}$ & $\begin{array}{l}1.0274 x \\
10^{5}\end{array}$ & 3446.42 & 3572.63 \\
\hline $\begin{array}{l}\text { Relative } \\
\text { Viscosity }\left(\mathrm{ms}^{-1}\right)\end{array}$ & 81.37 & 60.00 & 74.25 & 52.33 \\
\hline $\begin{array}{l}\text { Wall shear } \\
\text { stress }(\mathrm{Pa}) \\
\text { Specifica Heat }\end{array}$ & 0.001637 & 0.0017268 & 0.000992 & 0.000987 \\
\hline $\begin{array}{l}\text { Transfer } \\
\text { Coefficient } \\
\left(\mathrm{Wm}^{-1}-\mathrm{K}\right)\end{array}$ & 565.14 & 590.42 & $\mathrm{~N} / \mathrm{A}$ & $\mathrm{N} / \mathrm{A}$ \\
\hline $\begin{array}{l}\text { Total Enthalpy } \\
\left(\mathrm{JKg}^{-1}\right) \\
\text { Total }\end{array}$ & $\begin{array}{l}1.368 x \\
10^{6}\end{array}$ & $\begin{array}{l}1.5720 x \\
10^{6}\end{array}$ & $\mathrm{~N} / \mathrm{A}$ & $\mathrm{N} / \mathrm{A}$ \\
\hline $\begin{array}{l}\text { Temperature } \\
\text { (K) }\end{array}$ & 1159.70 & 1296.61 & $\mathrm{~N} / \mathrm{A}$ & $\mathrm{N} / \mathrm{A}$ \\
\hline
\end{tabular}



Figure 13(a): Pressure comparison for flat and dome head pistons



Figure 13 (b) Cell relative velocity comparison 




Figure 3(c): Specific Heat transfer coefficient



Figure 13(d): Turbulent velocity



Figure 14: Turbulent Kinetic Energy of flat head piston

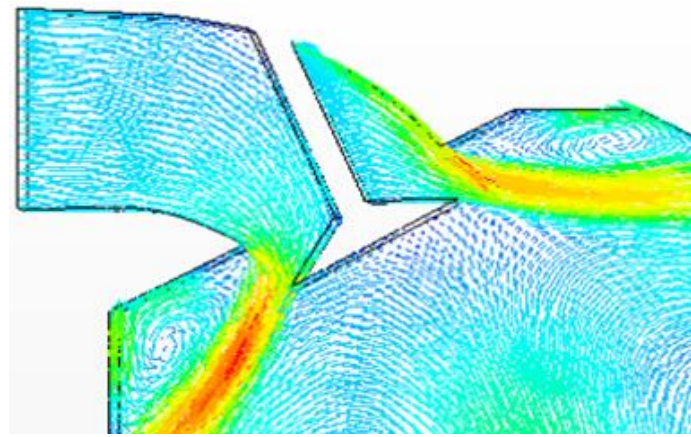

Figure 15: Divergence of air flow into the combustion chamber

The divergence phenomenon caused vortex shedding at the tips of the valve as shown in figure 15. This vortex shedding causes circulation of the fluid which results to a swirl. A three dimensional full model of the combustion chamber can display a swirl correctly. The high velocity at the constriction at the intake port can improve turbulence required for proper combustion process. Therefore, in optimizing the combustion chamber design, valve type can play a major role in optimizing the combustion process. This method is usually referred to as swirl management.

In conclusion, the design parameters used to optimize the air-fuel intake into the combustion chamber include cylinder head, intake manifold design and dimensions, valve diameter etc. Cylinder head design as presented in this model is a $30^{\circ}$ wedge type. As visualized in the postprocessed output, the wedge design created small vortices about the angled tip location which resulted into low velocities at those positions. This readily produced an increased pressure at the positions thus cooling the air-fuel mixture. An advantage of cooled air is the increase in density of the fuel mixture and on the other hand over cooling could possibly cause poor combustion resulting into the high formation of hydrocarbons and carbon monoxide instead of $\mathrm{CO}_{2}$ and steam.
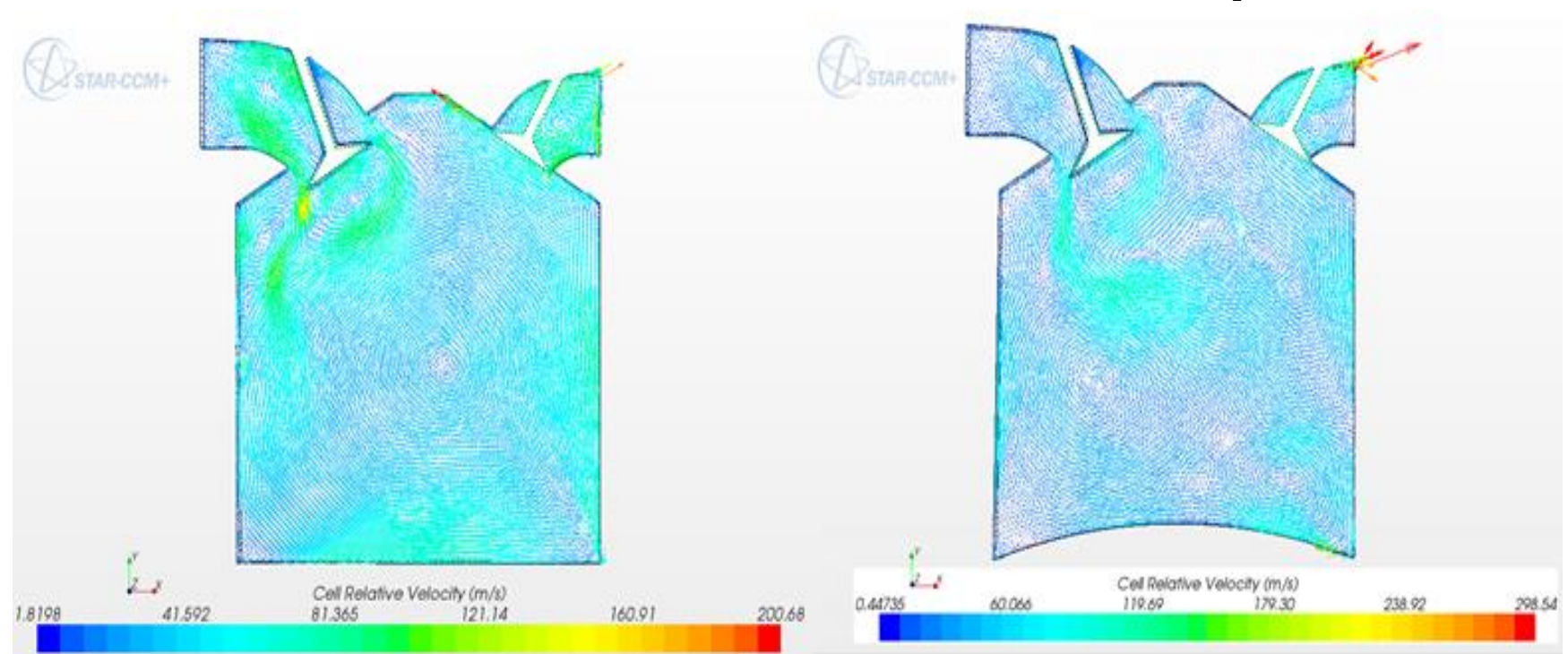

Figure 16: Comparison between the Relative Velocity of flat and dome pistons 
However, STAR-CCM V8 presented good visual display of the analysis and parameters with their values and also the corresponding distributions of the parameters within the combustion chamber. The optimized parameters extracted were similar to the CFD experiment performed by Ugur Kesgin [3] where he used a similar static model for the design of and inlet and exhaust system of an internal combustion engine. Also, the velocity magnitude visualization by Stefan's experiment [4] with OPEN Foam software showed similar characteristics with that of STAR-CCM. This demonstrates that similar results are possible even with different software. The analysis with STAR-CCM could be computationally expensive as it can take long hours for the solution to converge especially for volumetric mesh with smaller base size.

\section{ACKNOWLEDGEMENTS}

I acknowledge CD-Adapco for this expressive software. My regards also goes to the University of Hertfordshire, Aerospace Engineering Department for the opportunity to carry out this extensive research. I am not forgetting my family, co-authors, fellow colleagues and this journal.

\section{REFERENCES}

[1] H. Heisler, Advanced Engine Technology, Woburn: Buterworth-Heinemann, 2002.

[2] E. A. Mark Fogleman, "Application of the proper orthogonal decomposition to datasets of internal combustion engine flows," $T \& F$ Online Journal of TurbulenceVol. 5, pp. 1-23, 2004.

[3] U. Kesgin, "Study on the design of inlet and exhaust system of a stationary internal combustion engine," Energy Conversion and Management, Vol. 46, No. 13-14, p. 2258-2287, 2005.

[4] S. Gundmalm, "CFD modeling of a four stroke S. I. engine," KTH Industrial Engineering and Management, Stockholm, 2009.

[5] E. A. F. Duchaine, "Computational-Fluid-DynamicsBased Kriging" CERFACS, 42 Av. G. Coriolis, Toulouse, France, 2005.

[6] E. A. Pratima S. Patil, "Analysis of Internal Combustion Engine Heat Transfer Rate to Improve Engine Efficiency, Specific Power and Combustion Performance Prediction," International Journal of Mechanical Engineering and Technology, vol. 3, no. 2, pp. 447-452, 2012

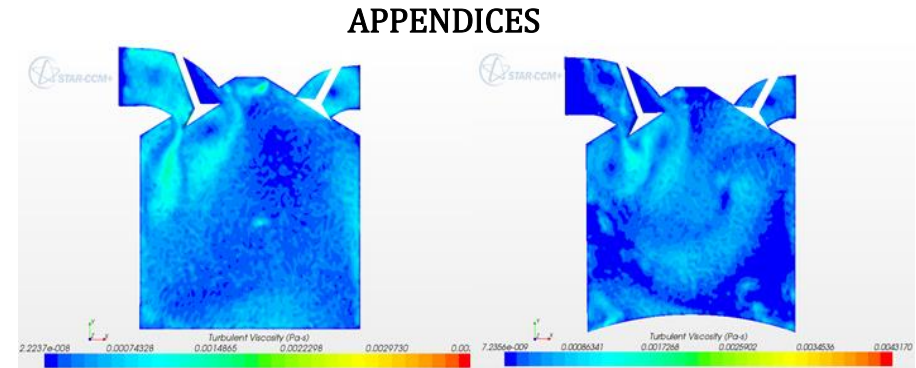

Figure A1: Comparison between the Turbulent Viscosity of flat and dome pistons

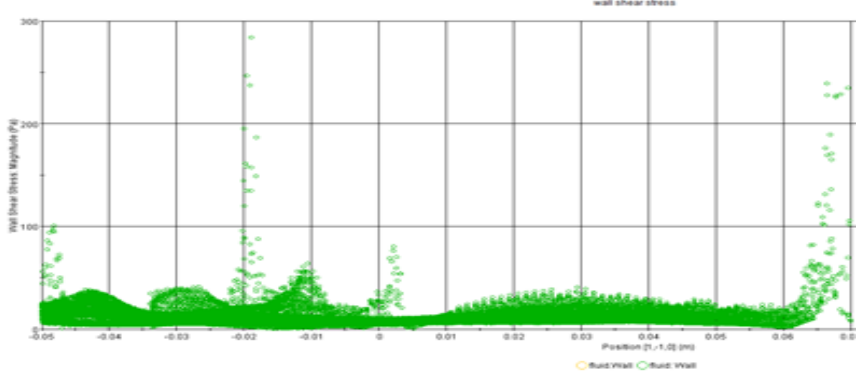

Figure A2: Wall shear stress across piston boundary layer (flat head piston)

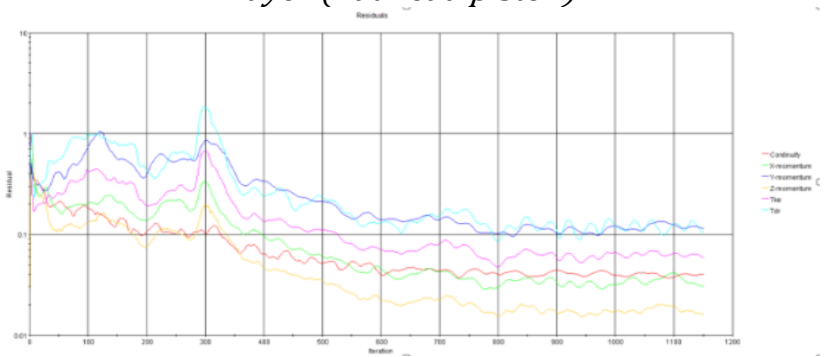

Figure A3: Convergence of computational results

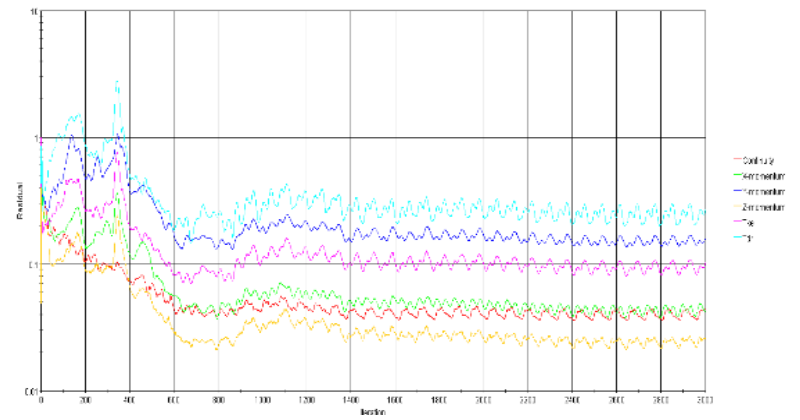

Figure A4: Monitoring efficiency performance of the intake stroke



Figure A5: Velocity magnitude visualization by Stefan Gundmalm using Open FOAM [4] 